\title{
Analysis of Peak to Average Power Ratio Reduction Techniques in Sfbc Ofdm System
}

\author{
T.Malathi ${ }^{1}$, A.Sasipriya ${ }^{2}$, S.Saravanan ${ }^{3}$ \\ ${ }^{I}$ Asst.Prof., Muthayammal Engineering College, Rasipuram. \\ ${ }^{2}$ Asst.Prof., Muthayammal Engineering College, Rasipuram \\ ${ }^{3}$ Prof., Muthayammal Engineering College, Rasipuram.
}

\begin{abstract}
Orthogonal Frequency Division Multiplexing (OFDM) has become the most popular modulation echnique for high speed data transmission. But the great disadvantage of the OFDM technique is its high Peak to Average Power Ratio (PAPR). In this paper, the Selected Mapping (SLM) technique and Clipping and Differential Scaling is applied to Space Frequency Block Coded (SFBC) OFDM systems to reduce the PAPR with Alamouti coding scheme. In SLM technique, different representations of OFDM symbols are generated by rotation of the original OFDM frame by different phase sequences, and the signal with minimum PAPR is selected and transmitted. To compensate for the effect of the phase rotation at the receiver, it is necessary to transmit the index of the selected phase sequence as side information (SI). Additionally, a suboptimum detection method that does not need SI is introduced at the receiver side. In Clipping and Differential Scaling technique, the amplitude of complex OFDM signal is clipped and then scaled in such a way so that the PAPR is reduced without causing much degradation in bit error rate (BER). The threshold values for clipping and scaling is determined using Monte Carlo Simulations. Simulation results show that the SLM method and Clipping and Scaling method effectively reduce the PAPR.
\end{abstract}

Keywords: Orthogonal frequency division multiplexing (OFDM), peak to average power ratio (PAPR), selected mapping (SLM), space frequency block coded (SFBC), Side information (SI), high power amplifiers (HPA), complementary cumulative density function (CCDF), inter-symbol interference (ISI).

\section{Introduction}

Orthogonal Frequency Division Multiplexing (OFDM) has been recently seen rising popularity in wireless applications. For wireless communications, an OFDM-based system can provide greater immunity to multi-path fading and reduce the complexity of equalizers. Now OFDM have been included in digital audio/video broad-casting (DAB/DVB) standard in Europe, and IEEE 802.11, IEEE 802.16 wireless broadband access systems, etc. The basic principle of OFDM is to split a high-rate data stream into a number of lower rate streams that are transmitted simultaneously over a number of subcarriers. These subcarriers are overlapped with each other. Because the symbol duration increases for lower rate parallel subcarriers, the relative amount of dispersion in time caused by multipath delay spread is decreased. Inter-symbol interference (ISI) is eliminated almost completely by introducing a guard time in every OFDM symbol.

One of the main drawbacks of OFDM is its high Peak to Average Power Ratio (PAPR) because it is inherently made up of so many subcarriers. The subcarriers are added constructively to form large peaks. High peak power requires High Power Amplifiers (HPA), A/D and D/A converters. Peaks are distorted nonlinearly due to amplifier imperfection in HPA. If HPA operates in nonlinear region, out of band and in-band spectrum radiations are produced which appears as the adjacent channel interference. Moreover if HPA is not operated in linear region with large power backs-offs, it would not be possible to keep the out-of-band power below the certain limits. This further leads to inefficient amplification and expensive transmitters. To prevent all these problems, power amplifiers has to be operated in its linear region. To overcome this problem, some algorithms have been proposed, which reduce the PAPR of the baseband OFDM signal [2]-[12]. Some of these methods need side information (SI) to be transmitted to the receiver, such as Partial Transmit Sequence (PTS) [8] and Selected Mapping (SLM) [9]. Some other methods do not need SI, such as clipping and filtering [5], tone reservation [6], [11], Polyphase Interleaving and Inversion [7] and Active Constellation Extension [2].

\section{Papr Of An Ofdm Signal}

Let us denote the data block of length $N$ as a vector $\mathrm{X}=\left[X_{0}, X_{1}, \ldots, X_{N-1}\right]^{T}$ where $N$ is equal to the number of sub-carriers. The duration of a symbol $X_{n}$ in X is $T$. Each symbol in X modulates one of a set of subcarriers, $\left\{f_{n}, n=0,1, \ldots, N-1\right\}$. The $N$ sub-carriers are chosen to be orthogonal, that is, $f_{n}=n \Delta f$, where $\Delta f=1 / N T$ and $N T$ is the duration of an OFDM data block $\mathrm{X}$. The complex envelope of the transmitted OFDM signal is given by 


$$
\mathrm{x}(\mathrm{t})=\frac{1}{\sqrt{N}} \sum_{k=0}^{N-1} X_{k} e^{j 2 \pi f_{k} t}, 0 \leq \mathrm{t} \leq \mathrm{NT}
$$

Presence of large number of independently modulated sub-carriers in an OFDM system the peak value of the system can be very high as compared to the average of the whole system. This ratio of the peak to average power value is termed as Peak-to-Average Power Ratio. Coherent addition of $\mathrm{N}$ signals of same phase produces a peak which is $\mathrm{N}$ times the average signal. The PAPR of the transmitted signal in (1) is defined as

$$
\operatorname{PAPR}(\mathrm{x})=\frac{\max n \mid\left\{\left.(n)\right|^{2}\right\}}{E\left\{|x(n)|^{2}\right\}}
$$

In principle, PAPR reduction techniques are concerned with reducing max $|\mathrm{x}(\mathrm{t})|$. The Cumulative Distribution Function (CDF) is one of the most regularly used parameters, which is used to measure the efficiency of any PAPR technique.

\section{Space Frequency Block Coded Orthogonal Frequency Division Multiplexing System} Model

In SFBC-OFDM systems with two transmitter antennas, the frequency-domain vector transmitted from the $p$ th antenna is denoted by

$$
\mathrm{X} p=[X p(0), X p(1), \ldots, X p(N c-1)]
$$

It is noteworthy that the orthogonality of the space frequency matrix $\mathbf{C}$ leads to full diversity at the receiver side. We have the space frequency matrix as follows. In this form will be the SFBC frames are made for transmitting by two transmitter antennas. We convert a normal BPSK signal to a SFBC signal for two transmitter ntennas.

$$
\begin{array}{lll}
\text { input_bpsk1(1) } & =\text { input_bpsk(1); } \\
\text { input_bpsk1(2) }= & \text {-conj(input_bpsk(2)); } \\
\text { input_bpsk2(1) } & \text { input_bpsk(2); } \\
\text { input_bpsk2(2) } & \text { conj(input_bpsk(1)) }
\end{array}
$$

The sfbc inputs are as follows input_bpsk1(1) is the first input to the first antenna, input_bpsk1(2) is the first input to the second antenna input_bpsk2(1) is the second input to the first antenna and input_bpsk2(1) is the second input to the second antenna. This can be represent in matrix form as follows

$$
\left(\begin{array}{ll}
X 1(2 v) & X 1(2 v+1) \\
X 2(2 v) & X 2(2 v+1)
\end{array}\right)=\left(\begin{array}{cc}
X(2 v) & X(2 v+1) \\
X *(2 v+1) & -X *(2 v)
\end{array}\right)
$$

where $v=0,1, \ldots, \mathrm{Nc} / 2-1$.

The vectors $\mathrm{X} 1$ and $\mathrm{X} 2$ can be generated from the original OFDM frame $\mathrm{X}$. The vectors $\mathrm{X} 1$ and $\mathrm{X} 2$ are passed through the IFFT operation to yield the time-domain samples $x_{1}(n)$ and $x_{2}(n), 0 \leq n \leq N-1$. It is noteworthy that the orthogonality of the space frequency matrix $\mathbf{C}$ in (4) leads to full diversity at the receiver side, i.e.,

$$
\mathbf{C C}^{\mathrm{H}}=|\mathrm{X}(2 v)|^{2}+|\mathrm{X}(2 v+1)|^{2} \mathbf{I}_{2}
$$

where $\mathbf{I}_{\mathrm{n}}$ is the $n \times n$ identity matrix. The PAPR of the $p$ th antenna is defined by

$$
\operatorname{PAPR}\left\{\mathrm{x}_{\mathrm{p}}\right\}=\frac{\left.\max n|\min (n)|^{2}\right\}}{E\left\{|x p(n)|^{2}\right\}} \quad p=1,2
$$

where $E\{$.$\} is the mathematical expectation. The overall PAPR of the SFBC-OFDM system is defined by$

$$
\mathrm{PAPR}=\max _{\mathrm{p} \in\{1,2\}} \operatorname{PAPR}\left\{\mathbf{x}_{\mathrm{p}}\right\}
$$

\section{Selected Mapping For Peak-To-Average Power Ratio Reduction Of Space-Frequency Block Coded (Ofdm) Systems}

In this paper, it is shown that the simplified SLM technique can be applied to SFBC-OFDM systems with two transmitter antennas and Almouti coding scheme without changing the orthogonality of space frequency coding. In this method, the optimum phase sequence is applied to the OFDM frames of two antennas such that the SFBC structure remains constant. Then, it will be shown that, at the receiver side, the optimum phase sequence can be detected without SI. Detection of phase sequence index does not need any extra transmission power or extra computation complexity of the transmitter and only uses the intrinsic redundancy of the SFBC code. 


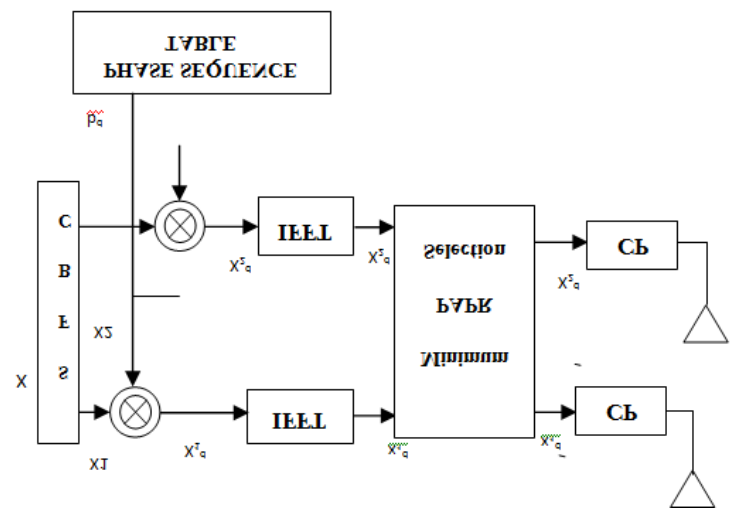

Fig.1. Block diagram of SFBC OFDM transmitter with two transmitter antennas and with SLM method for PAPR reduction.

The Block diagram of SFBC OFDM transmitter with two transmitter antennas and with SLM method for PAPR reduction is shown in the Fig 1.Vectors X1 and X2 can be multiplied by $D$ different phase sequences to yield the minimum PAPR representation, but the SFBC structure must remain constant, so that full diversity can be achieved. In simplified SLM for PAPR reduction of spatial multiplexed OFDM has been proposed. In this scheme, the OFDM frames of the antennas are simultaneously modified with the same single-phase sequence. This leads to reduction in the number of bits that must be transmitted as SI.

In this paper, this approach is used for SFBC-OFDM systems. It is shown that, if this method is used for the SFBC-OFDM system, then the intrinsic redundancy of space frequency coding can be used to detect the index of the phase sequence at the receiver side without SI. Based on this approach, $D$ different representations of the signals $\mathrm{x} 1$ and $\mathrm{x} 2$ are generated.

$$
\begin{aligned}
& \mathrm{X}_{1}^{\mathrm{d}}=\operatorname{IFFT}_{\mathrm{N}}\left\{\mathrm{X}_{1} \otimes \mathrm{b}^{\mathrm{d}}\right\} \\
& \mathrm{X}_{2}^{\mathrm{d}}=\operatorname{IFFT}_{\mathrm{N}}\left\{\mathrm{X}_{2} \otimes \mathrm{b}^{\mathrm{d}}\right\}, \quad 0 \leq \mathrm{d} \leq \mathrm{D}-1
\end{aligned}
$$

It has been shown that a simple and optimal choice for the phase sequences is a random selection of 0 and $\pi$ with equal probabilities. In this case, the complexity of the phase rotation is very low, because $b^{d}(k)=e j 0$ $=+1$ or $b^{d}(k)=e j \pi=-1$; thus, the multiplication of the frequency-domain vectors by the vector $\mathrm{b}^{d}$ is via the sign change of some of the symbols.

By keeping the space frequency code orthogonal, full diversity can be achieved at the receiver side. Consider an OFDM consisting of $N$ subcarriers. Let a block of $N$ symbol $\mathrm{X}=\mathrm{XK}, \mathrm{k}=0,1,2, \ldots, \mathrm{N}-1$ is formed with each symbol modulating one of a set of subcarriers. $\left\{f_{k}, \mathrm{k}=0,1,2, \ldots \mathrm{N}-1\right\}$. The $N$ subcarriers are chosen to be orthogonal, that is, $f_{k}=k \Delta f$, where $\Delta f=\frac{1}{N T}$ and $\mathrm{T}$ is the original symbol period. Therefore, the complex baseband OFDM signal can be written as

$$
\mathrm{x}(\mathrm{t})=\frac{1}{\sqrt{N}} \sum_{k=0}^{N-1} X_{k} e^{j 2 \pi f_{k} t}, 0 \leq \mathrm{t} \leq \mathrm{NT}
$$

In general, the PAPR of OFDM signal is defined as the ratio period between the maximum instantaneous power and its average power during an OFDM symbol.

$$
\text { PAPR }=\frac{\max _{0 \leq t \leq N T}\left[|x(t)|^{2}\right]}{1 /(N T) \int_{0}^{N T}|x(t)|^{2} d t}
$$

Reducing the $\max |x(t)|$ is the principle goal of PAPR reduction techniques. In practice, most systems deal with a discrete-time signal, therefore, we have to sample the continuous-time signal $x(t)$.

To better approximate the PAPR of continuous-time OFDM signals, the OFDM signals samples are obtained by times oversampling. By sampling $\mathrm{x}(\mathrm{t})$ defined in Equation, at frequency $f_{s}=L / T$, wher $L$ is the oversampling factor, the discrete-time OFDM symbol can be written as

$$
\mathrm{x}(\mathrm{n})=\frac{1}{\sqrt{N}} \sum_{k=0}^{N-1} X_{k} e^{j \frac{2 \pi}{N L} k n}, 0 \leq \mathrm{n} \leq \mathrm{NL}
$$

Equation (11) can be implemented by using a length (NL) IFFT operation. The new input vector $X$ is ex-tended from original $X$ by using the so-called zero-padding scheme, i.e., by inserting zeros in the middle of $X$. The PAPR computed form the $L$-times oversampled time domain OFDM signal samples can be defined as 


$$
\operatorname{PAPR}[x(n)]=10 \log \frac{\max _{0 \leq t \leq N L-1}\left[|x(n)|^{2}\right]}{E\left[|x(n)|^{2}\right]}
$$

We can evaluate the performance of PAPR using the cumulative distribution of PAPR of OFDM signal. The cumulative distribution function (CDF) is one of the most regularly used parameters, which is used to measure the efficiency of and PAPR technique. The CDF of the amplitude of a signal sample is given by

$$
\mathrm{F}(\mathrm{z})=1-\exp (\mathrm{z})
$$

However, the complementary CDF (CCDF) is used instead of CDF, which helps us to measure the probability that the PAPR of a certain data block exceeds the given threshold. The CCDF of the PAPR of the data block is desired is our case to compare outputs of various reduction techniques. This is given by

$$
\begin{aligned}
\mathrm{P}(\mathrm{PAPR}>\mathrm{z}) & =1-\mathrm{P}(\mathrm{PAPR}>\mathrm{z}) \\
& =1-(\mathrm{F}(\mathrm{z}))^{\mathrm{N}} \\
& =1-(1-\exp (-\mathrm{z}))^{\mathrm{N}}
\end{aligned}
$$

\section{Clipping And Differential Scaling}

A new technique called Clipping and Differential Scaling is proposed. The probability distribution of amplitudes of the OFDM signal follows Rayleigh distribution and thus the probability of high peaks is very less. Select the clipping threshold at which the BER is degraded from $1.5 \times 10-3$ to $3.5 \times 10-3$ at SNR of $10 \mathrm{~dB}$ and the amplitudes above this clipping threshold are clipped. Differential Scaling is used to reduce the PAPR but not deteriorate the BER. Since different ranges of amplitudes of the signal are scaled in a different manner, it is called Differential Scaling. The three types of scaling are described below.

\section{A. Scale Up}

In this method, scale up the lower amplitudes of the signal by a factor of $\beta$. This leads to increase the average value without affecting the peak values. Therefore, the resulting PAPR reduces. The PAPR reduction function can be defined as

$$
\begin{aligned}
h(x) & =\alpha x_{p}, \text { if } x>\alpha x_{p} \\
& =\beta x, \text { if } x<A \\
& =x, \text { if } A \leq x \leq \alpha x_{p}
\end{aligned}
$$

where $x_{p}$ is the amplitude peak value occurring in an OFDM symbol block, $\alpha$ is the factor deciding the clipping threshold in terms of percentage of the peak value and $\beta$ is the scaling factor for the range $[0, A)$ whose value is greater than one. The values of the parameters used are mentioned at the end of this section.

B. Scale Down

In this method, scale down the higher amplitudes of the signal by a factor of $\gamma$. This leads to decrease the peak value. Although the average value would also fall down, the resulting PAPR reduces. Because the reduction in peak power is greater than the reduction in the average power. The PAPR reduction function can be defined as

$$
\begin{aligned}
h(x) \quad & =\alpha x_{p}, \text { if } x>\alpha x_{p} \\
& =\gamma x, \text { if } B \leq x \leq \alpha x_{p} \\
& =x, \text { if } x<B
\end{aligned}
$$

where $x_{p}$ is the amplitude peak value occurring in an OFDM symbol block, $\alpha$ is the factor deciding the clipping threshold in terms of percentage of the peak value and $\gamma$ is the scaling factor for the range $\left[\beta, \alpha x_{p}\right]$ whose value is less than one. The values of the parameters used are mentioned at the end of this section.

C. Scale Up and Down

In this method, combine both the above-mentioned approaches i.e. up-scaling and down-scaling. This method exploits the advantages of both the methods. Hence, a PAPR can be reduced considerably. The PAPR reduction function can be defined as

$$
\begin{aligned}
h(x) \quad & =\alpha x_{p}, \text { if } x>\alpha x_{p} \\
& =\gamma x, \text { if } B \leq x \leq \alpha x_{p} \\
& =\beta x, \text { if } x<A
\end{aligned}
$$

\section{Simulation Result}

In this section, simulation results for the proposed techniques are presented. The simulation is based on the system model in Fig. 1. The reduction of PAPR by Selected Mapping technique is shown in Fig.2. 


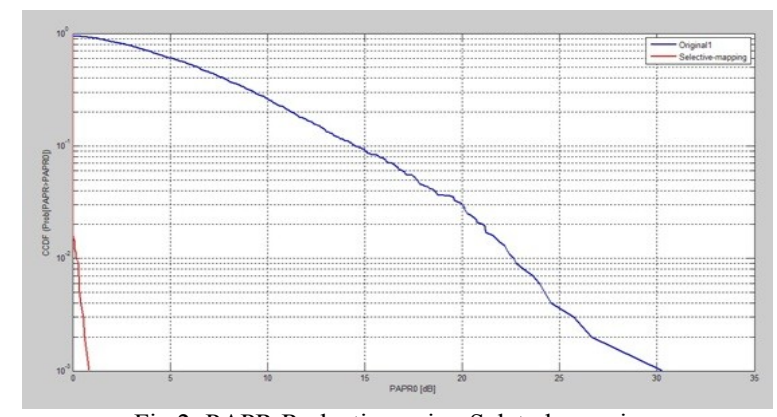

Fig.2. PAPR Reduction using Seleted mapping

The original signal has the PAPR value of nearly $65 \mathrm{db}$. The Clipping and Scaling Technique is applied to that signal that is Scale Up, Scale Down and Scale Up Down techniques are applied.
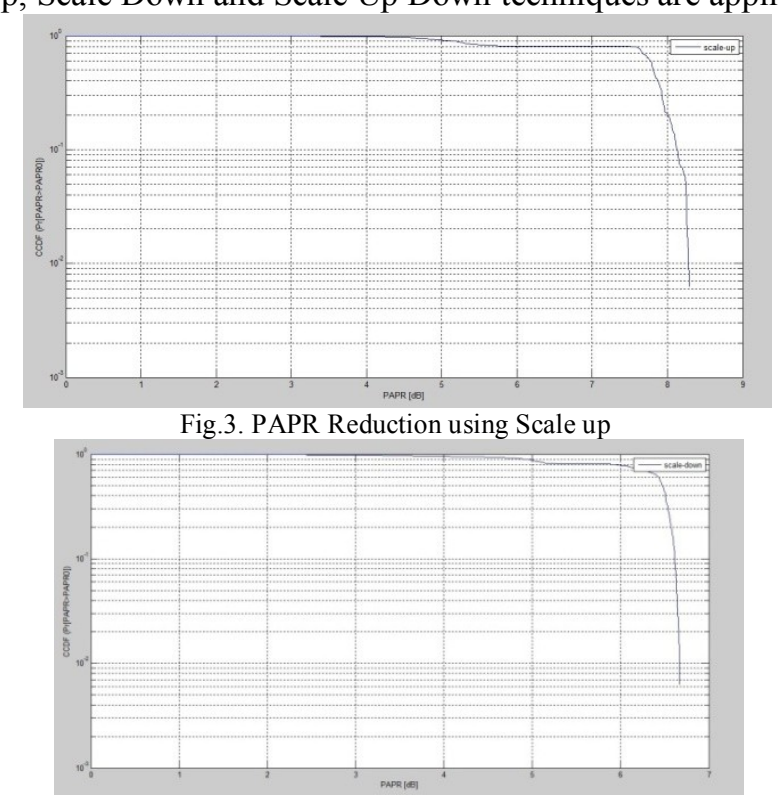

Fig.4. PAPR Reduction using Scale down

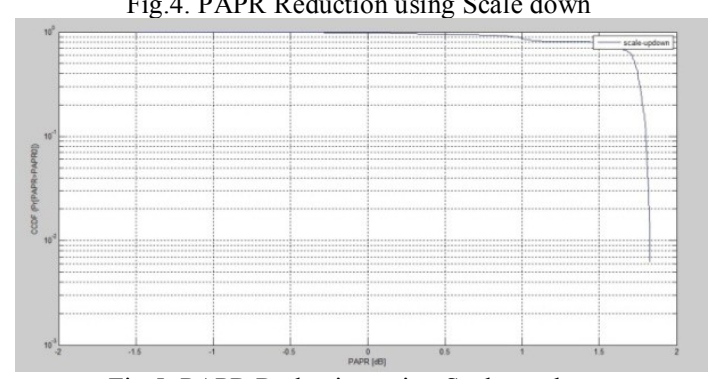

Fig.5. PAPR Reduction using Scale up down

The Scale Up Technique is applied to that signal, the PAPR value is reduced to nearly $8 \mathrm{db}$. The Scale Down Technique is applied to that signal, the PAPR value is reduced to nearly $6.5 \mathrm{db}$. The Scale Up Down Technique is applied to that signal, the PAPR value is reduced to nearly $1.5 \mathrm{db}$. Among these three techniques, the Scale Up Down Technique is the best method to reduce the PAPR value. The Scale Up, Scale Down and Scale Up Down method is described separately in Fig.3., Fig.4., and Fig.5. Fig. 6 shows the CCDF with Scale up, Scale own and Scale up-down by Clipping and Differential scaling.

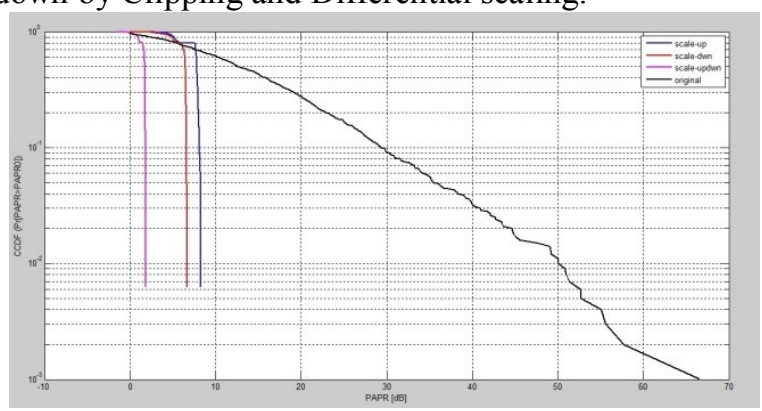

Fig.6. PAPR Reduction using Clipping and Differential Scaling 


\section{Conclusion}

In this paper, a novel PAPR reduction technique based on Selected Mapping Technique is done. In this method, the same phase sequence is concurrently applied to the frequency-domain signals for both antennas, and the signal with minimum PAPR has been found and transmitted. A low-complexity suboptimum detector has been proposed to detect the phase sequence index without SI. A simple approach based on Clipping and Differential Scaling is proposed to reduce the PAPR of OFDM signals. Clipping along with three different scaling methods, namely up-scaling, down-scaling, up-down scaling is described. Simulation results show that the performance of the Selected Mapping method and Clipping and Differential Scaling in PAPR reduction of the SFBC OFDM system with two transmitter antennas for BPSK modulation.

\section{REFERENCES}

[1] Hwang.T, Yang.C, Wu.G, Li.S, and Lee.G.Y, “OFDM and its wireless application: A survey,” IEEE Trans. Veh. Technol., vol. 58, no. 4, pp. 1673-1694, May 2009.

[2] Bae.K, Andrews.J.G, and Powers.E.J, "Adaptive active constellation extension algorithm for peak-to-average ratio reduction in OFDM,” IEEE Commun. Lett., vol. 14, no. 1, pp. 39-41, Jan. 2010.

[3] Jiang.T and Li.X, "Using fountain codes to control the peak-to- average power ratio of OFDM signals," IEEE Trans. Veh. Technol., vol. 59, no. 8, pp. 3779-3785, Oct. 2010.

[4] Jie.Y, Lei.C, and De.C, "A modified selected mapping technique to reduce the peak-to average power ratio of OFDM signal," IEEE Trans. Consum. Electron., vol. 53, no. 3, pp. 846 851, Aug. 2007.

[5] Kwon.U.K, Kim.D, and Im.G.H, "Amplitude clipping and iterative reconstruction of MIMO-OFDM signals with optimum equalization,” IEEE Trans. Wireless Commun., vol. 8, no. 1, pp. 268-277, Jan. 2009.

[6] Krongold.B.S and Jones.D.L, "An active-set approach for OFDM PAR reduction via tone reservation," IEEE Trans. Signal Process., vol. 52, no. 2, pp. 495-509, Feb. 2004.

[7] Lati.novic.Z and Bar-Ness.Y, "SFBC MIMO-OFDM peak-to-average power ratio reduction by polyphase interleaving and inversion," IEEE Commun. Lett., vol. 10, no. 4, pp. 266-268, Apr. 2006.

[8] Lu.G, Wu.P, and Carlemalm-Logothetis.C, "Peak-to-average power ratio reduction in OFDM based on transformation of partial transmit sequences,” Electron. Lett., vol. 42, no. 2, pp. 105-106, Jan. 2006.

[9] Mahmoud Ferdosizadeh Naeiny and Farokh Marvasti, "Selected Mapping Algorithm for PAPR Reduction of Space-Frequency Coded OFDM System without Side Information," IEEE Trans. Veh. Techno., Vol. 60, no. 3, Mar 2011

[10] Wang.S.H and Li.C.P, "A low-complexity PAPR reduction scheme for SFBC MIMO-OFDM systems," IEEE Signal Process. Lett., vol. 16, no. 11, pp. 941-944, Nov. 2009.

[11] Wang.L and Tellambura.C, "Analysis of clipping noise and tone reservation algorithms for peak reduction in OFDM systems," IEEE Trans. Veh. Technol., vol. 57, no. 3, pp. 1675-1694, May 2008.

[12] Young-Jin Kim, Ui-Kun Kwon, Dae-Young Seol, and Gi-Hong Im, "An Effective PAPR Reduction of SFBC-OFDM for Multinode Cooperative Transmission” IEEE Trans. Signal Process., vol. 16, no. 11, pp., Nov. 2009 